\title{
Correction to: Pattern Recognition and Computer Vision
}

Huimin Ma D, Liang Wang, Changshui Zhang, Fei Wu $\mathbb{D}$,

Tieniu Tan, Yaonan Wang, Jianhuang Lai, and Yao Zhao (D)

\section{Correction to: \\ H. Ma et al. (Eds.): Pattern Recognition and Computer Vision, LNCS 13020, https://doi.org/10.1007/978-3-030-88007-1}

\author{
For Chapter 3: \\ An acknowledgement for a grant from the National Natural Science Foundation of \\ China for the paper has been added. \\ For Chapter 11: \\ A co-author (Shiping Wang) was not included in the original publication due to an \\ oversight. This co-author has been added in this correction, reflecting the original \\ submission of the article.
}

The updated version of these chapters can be found at https://doi.org/10.1007/978-3-030-88007-1_3

https://doi.org/10.1007/978-3-030-88007-1_11

(C) Springer Nature Switzerland AG 2021

H. Ma et al. (Eds.): PRCV 2021, LNCS 13020, p. C1, 2021.

https://doi.org/10.1007/978-3-030-88007-1_55 